\title{
Molecular Interaction Study of Mixture of $N, N$-Dimethylformamide, Cyclohexane and Pyridine at Different Frequencies
}

\author{
MANOJ KU. PRAHARAJ ${ }^{\mathrm{a}, *}$, ABHIRAM SATAPATHY $^{\mathrm{b}}$, \\ PRATIVARANI MISHRA $^{\mathrm{b}}$ and SARMISTHA MISHRA ${ }^{\mathrm{b}}$
}

${ }^{\text {a } P . G . D e p a r t m e n t ~ o f ~ P h y s i c s, ~ R a v e n s h a w ~ U n i v e r s i t y, ~ C u t t a c k, ~ O d i s h a ~-753003, ~ I n d i a ~}$

${ }^{\mathrm{b}}$ Department of Physics, ABIT, CDA, Sector-1, Cuttack, Odisha- 753014, India

abitphysics@rediffmail.com

Received 4 January 2013 / Accepted 14 February 2013

\begin{abstract}
The ultrasonic velocity, density and viscosity have been measured for ternary mixture of $N, N$ dimethylformamide (DMF), cyclohexane and pyridine at different frequencies $2 \mathrm{MHz}, 4 \mathrm{MHz}$ and $6 \mathrm{MHz}$ but at constant temperature $318 \mathrm{~K}$. These experimental data's have been used to estimate the parameters such as adiabatic compressibility $(\beta)$, intermolecular free length $\left(\mathrm{L}_{\mathrm{f}}\right)$, Gibbs' free energy $(\Delta G)$, free volume $\left(V_{f}\right)$, specific acoustic impedance $(Z)$ and absorption coefficient $\left(\alpha / f^{2}\right)$ for the mixture. The excess values of these parameters are also evaluated and the molecular associations in the mixture are discussed.
\end{abstract}

Keywords: Ultrasonic velocity, Adiabatic compressibility, Free length, Free volume, Gibbs free energy

\section{Introduction}

Ultrasonic velocity measurements have been successfully employed to detect and assess weak and strong molecular interactions present in binary ${ }^{1-3}$ and ternary ${ }^{4-6}$ liquid mixtures. Such studies lead to the determination of acoustic and thermodynamic parameters of liquid mixture. Their dependence on concentration, temperature and frequency are of importance in many fields of applied research and also find applications in many important chemical, industrial and biological processes ${ }^{7}$.

The excess properties of the above parameters also play significant roles in the above studies as they are highly sensitive to molecular interactions in the mixture. We intend to measure the ultrasonic parameters and their excess values for ternary mixture of $N-N$ dimethylformamide and pyridine along with cyclohexane at different concentration of the components of the mixture and at different frequencies. We have done similar studies in liquid mixtures like DMF + chlorobenzene + cyclohexane and DMF + Nitrobenzene + Cyclohexane ${ }^{8}$. We have shown that the mixture containing chlorobenzene is more sensitive to the changes in frequency in comparison to the mixture containing nitrobenzene. 
Pyridine $\left(\mathrm{C}_{5} \mathrm{H}_{5} \mathrm{~N}\right)$ with nitrogen atom attached to one end of its structure is a polar aprotic solvent and negligibly basic. Pyridine is an important dipolar liquid used currently in extraction process for coal and to analyse its components. It is used in the manufacture of vitamin $\mathrm{B}_{6}$ and other drugs. DMF is a versatile solvent with a high dielectric constant and a high dipole moment. Cyclohexane has a closed chain structure and is non-polar in nature. Dispersion forces caused by correlated movements of electrons are possible between cyclohexane and the other components ${ }^{9-10}$.

\section{Experimental}

All the chemicals namely, pyridine, DMF and cyclohexane are obtained from E-Merck Chemicals Ltd., India. The velocity of ultrasonic waves is measured, using a multifrequency ultrasonic interferometer (Model M-84, supplied by M/s Mittal Enterprises, New Delhi). The measuring cell of interferometer is a specially designed double walled vessel. An electronically operated digital constant temperature bath (Model SSI-03spl, supplied by M/s Mittal Enterprises, New Delhi), with an accuracy of $\pm 0.1 \mathrm{~K}$ has been used to circulate water through the outer jacket of the double walled measuring cell containing the experimental liquid. The density is measured by a specific gravity bottle using the formula

$$
\rho_{2}=\left(w_{2} / w_{1}\right) \cdot \rho_{1}
$$

Where, $w_{1} \& w_{2}=$ weight of distilled water\& experimental liquid respectively. $\rho_{1} \& \rho_{2}=$ Density of water \& experimental liquid respectively.

Viscosity measurements were carried out with an Oswald's viscometer by using the relation.

$$
\eta_{2}=\eta_{1}\left(t_{2}, t_{1}\right)\left(\rho_{2} / \rho_{1}\right)
$$

Where, $\eta_{1}=$ Viscosity of water, $\eta_{2}=$ Viscosity of mixture, $\rho_{1}=$ Density of water, $\rho_{2}=$ Density of mixture, $t_{1}=$ Time of flow of water, $t_{2}=$ Time of flow of mixture.

The time of flow was measured using a digital racer stop watch with an accuracy of $0.1 \mathrm{sec}$.

\section{Theory}

Following relations ${ }^{11-14}$ are used to calculate the parameters by using the experimental values of density, viscosity and ultrasonic velocity.

$$
\begin{aligned}
\beta & =1 / \mathrm{U}^{2} \cdot \rho \\
\mathrm{L}_{\mathrm{f}} & =\mathrm{K}_{\mathrm{T}} \cdot \beta 1^{/ 2} \\
\Delta \mathrm{G} & =\mathrm{kT} \cdot \mathrm{Ln}(\mathrm{kT} \tau / \mathrm{h}) \\
\mathrm{V}_{\mathrm{f}} & =\left(\mathrm{M}_{\mathrm{eff}} \cdot \mathrm{U} / \mathrm{K} \cdot \eta\right)^{3 / 2} \\
\mathrm{Z} & =\mathrm{U} \cdot \rho \\
\alpha / \mathrm{f}^{2} & =8 \pi^{2} \eta / 3 \rho \mathrm{U}^{2}
\end{aligned}
$$

Where ' $\mathrm{K}_{\mathrm{T}}$ ' is the temperature dependent constant known as Jacobson's constant, ' $\mathrm{k}$ ' is Boltzmann's constant, ' $h$ ' is the Planck's constant, ' $\mathrm{b}$ ' stands for cubic packing, which is assumed to be ' 2 ' for all liquids, ' $R$ ' is gas constant, ' $T$ ' is absolute temperature, ' $M_{\text {eff }}$ ' is the effective mass of liquid mixture, ' $\mathrm{K}$ ' is a dimensionless constant independent of temperature and liquid, its value is $4.281 \times 10^{9}$.

The excess values of parameters has been calculated by using the relation

$$
A^{E}=A_{\text {exp }}-A_{i d}
$$

Where, $A_{i d}=\Sigma A_{i} X_{i}, i=1$ to $n, A_{i}$ is any acoustical parameters and $X_{i}$ is the mole fraction of the liquid component. 


\section{Results and Discussion}

The experimental values of density, viscosity and ultrasonic velocity for the ternary mixture are listed in Table 1. Values of different parameters for the mixture are given in the Table $2 \& 3$. The excess values of these parameters are given in Table $4 \& 5$. Variations of these parameters and their excess values with frequency are plotted in the Figure 1 to 11.

Table 1. Values of density, viscosity and velocity at $2 \mathrm{MHz}, 4 \mathrm{MHz}$ and $6 \mathrm{MHz}$

\begin{tabular}{cccccccccccc}
\hline \multirow{2}{*}{ Mole fraction } & \multicolumn{3}{c}{$\begin{array}{c}\text { Density }(\rho) \\
\text { Kg.m }\end{array}$} & \multicolumn{3}{c}{$\begin{array}{c}\text { Viscosity }(\eta) \\
10^{-3} \text { N.s.m }\end{array}$} & \multicolumn{3}{c}{$\begin{array}{c}\text { Velocity(U) } \\
\text { m.s }\end{array}$} \\
\hline $\mathrm{X}_{1}$ & $\mathrm{X}_{3}$ & $2 \mathrm{MHz}$ & $4 \mathrm{MHz}$ & $6 \mathrm{MHz}$ & $2 \mathrm{MHz}$ & $4 \mathrm{MHz}$ & $6 \mathrm{MHz}$ & $2 \mathrm{MHz}$ & $4 \mathrm{MHz}$ & $6 \mathrm{MHz}$ \\
0.0000 & 0.6000 & 869.56 & 869.56 & 869.56 & 0.571 & 0.571 & 0.571 & 1247.2 & 1238.4 & 1224.6 \\
0.0999 & 0.4999 & 864.92 & 864.92 & 864.92 & 0.599 & 0.599 & 0.599 & 1251.3 & 1242.6 & 1228.6 \\
0.1998 & 0.4001 & 861.20 & 861.20 & 861.20 & 0.612 & 0.612 & 0.612 & 1255.0 & 1246.1 & 1232.9 \\
0.3001 & 0.3000 & 858.42 & 858.42 & 858.42 & 0.630 & 0.630 & 0.630 & 1258.9 & 1248.8 & 1235.5 \\
0.4000 & 0.1999 & 854.71 & 854.71 & 854.71 & 0.643 & 0.643 & 0.643 & 1263.6 & 1252.1 & 1238.8 \\
0.4998 & 0.1001 & 851.00 & 851.00 & 851.00 & 0.660 & 0.660 & 0.660 & 1268.5 & 1255.4 & 1242.1 \\
0.5997 & 0.0000 & 846.23 & 846.23 & 846.23 & 0.670 & 0.670 & 0.670 & 1274.5 & 1260.6 & 1247.2 \\
\hline
\end{tabular}

Table 2. Values of adiabatic compressibility free length and free volume at $2 \mathrm{MHz}, 4 \mathrm{MHz}$ and $6 \mathrm{MHz}$

\begin{tabular}{|c|c|c|c|c|c|c|c|c|c|c|}
\hline \multicolumn{2}{|c|}{ Mole fraction } & \multicolumn{3}{|c|}{$\begin{array}{c}\text { Adiabatic } \\
\text { compressibility }(\beta) \\
\times 10^{-10} \mathrm{~N}^{-1} \cdot \mathrm{m}^{2}\end{array}$} & \multicolumn{3}{|c|}{$\begin{array}{l}\text { Free length }\left(\mathrm{L}_{\mathrm{f}}\right) \\
\quad \times 10^{-10} \mathrm{~m}\end{array}$} & \multicolumn{3}{|c|}{$\begin{array}{l}\text { Free volume }\left(\mathrm{V}_{\mathrm{f}}\right) \\
\times 10^{-7} \mathrm{~m}^{3} \cdot \mathrm{mol}^{-1}\end{array}$} \\
\hline $\mathrm{X}_{1}$ & $\mathrm{X}_{3}$ & $2 \mathrm{MHz}$ & $4 \mathrm{MHz}$ & $6 \mathrm{MHz}$ & $2 \mathrm{MHz}$ & $4 \mathrm{MHz}$ & $6 \mathrm{MHz}$ & $2 \mathrm{MHz}$ & $4 \mathrm{MHz}$ & $6 \mathrm{MHz}$ \\
\hline 0.0000 & 0.6000 & 7.3932 & 2.664 & 2.664 & 2.664 & 0.5562 & 0.5624 & 2.664 & 2.636 & 2.592 \\
\hline 0.0999 & 0.4999 & 7.3844 & 2.462 & 2.462 & 2.462 & 0.5558 & 0.5621 & 2.462 & 2.437 & 2.396 \\
\hline 0.1998 & 0.4001 & 7.3724 & 2.367 & 2.367 & 2.367 & 0.5554 & 0.5613 & 2.367 & 2.342 & 2.305 \\
\hline 0.3001 & 0.3000 & 7.3505 & 2.255 & 2.255 & 2.255 & 0.5551 & 0.5611 & 2.255 & 2.228 & 2.193 \\
\hline 0.4000 & 0.1999 & 7.3276 & 2.172 & 2.172 & 2.172 & 0.5548 & 0.5608 & 2.172 & 2.142 & 2.108 \\
\hline 0.4998 & 0.1001 & 7.3028 & 2.079 & 2.079 & 2.079 & 0.5546 & 0.5605 & 2.079 & 2.046 & 2.014 \\
\hline 0.5997 & 0.0000 & 7.2750 & 2.020 & 2.020 & 2.020 & 0.5538 & 0.5598 & 2.020 & 1.987 & 1.955 \\
\hline
\end{tabular}

Table 3. Values of Gibb's free energy, acoustic impedance and absorption coefficient at $2 \mathrm{MHz}, 4 \mathrm{MHz}$ and $6 \mathrm{MHz}$

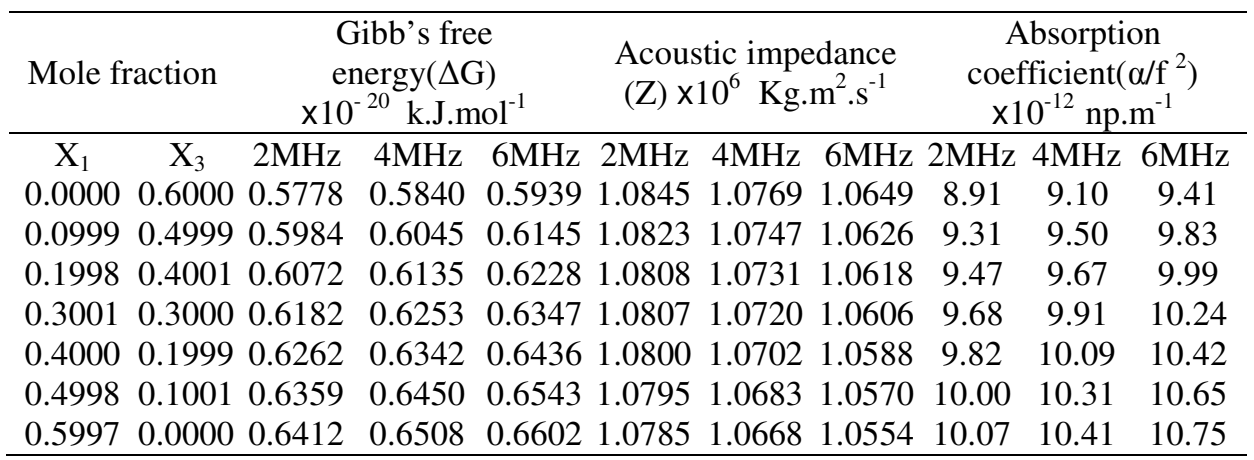


Table 4. Values of excess-adiabatic compressibility, excess free length and excess free volume at $2 \mathrm{MHz}, 4 \mathrm{MHz}$ and $6 \mathrm{MHz}$

\begin{tabular}{cccccccccccc}
\hline \multirow{2}{*}{ Mole fraction } & \multicolumn{3}{c}{$\begin{array}{c}\beta^{\mathrm{E}} \\
\times 10^{-10} \mathrm{~N}^{-1} \cdot \mathrm{m}^{2}\end{array}$} & \multicolumn{3}{c}{$\begin{array}{c}\mathrm{L}_{\mathrm{f}}^{\mathrm{E}} \\
\mathrm{x} 10^{-10} \mathrm{~m}\end{array}$} & \multicolumn{2}{c}{$\mathrm{x}^{-70^{-7}} \mathrm{~m}^{3} \cdot \mathrm{mol}^{-1}$} \\
\hline $\mathrm{X}_{1}$ & $\mathrm{X}_{3}$ & $2 \mathrm{MHz}$ & $4 \mathrm{MHz}$ & $6 \mathrm{MHz}$ & $2 \mathrm{MHz}$ & $4 \mathrm{MHz}$ & $6 \mathrm{MHz}$ & $2 \mathrm{MHz}$ & $4 \mathrm{MHz}$ & $6 \mathrm{MHz}$ \\
0.0000 & 0.6000 & 0.035 & 0.115 & 0.265 & 0.0060 & 0.0090 & 0.0145 & -0.450 & -0.470 & -0.508 \\
0.0999 & 0.4999 & 0.055 & 0.134 & 0.287 & 0.0070 & 0.0099 & 0.0155 & -0.570 & -0.588 & -0.623 \\
0.1998 & 0.4001 & 0.071 & 0.152 & 0.295 & 0.0077 & 0.0107 & 0.0159 & -0.585 & -0.602 & -0.633 \\
0.3001 & 0.3000 & 0.077 & 0.172 & 0.316 & 0.0080 & 0.0115 & 0.0168 & -0.616 & -0.635 & -0.666 \\
0.4000 & 0.1999 & 0.084 & 0.194 & 0.338 & 0.0085 & 0.0126 & 0.0179 & -0.618 & -0.640 & -0.670 \\
0.4998 & 0.1001 & 0.087 & 0.216 & 0.360 & 0.0088 & 0.0135 & 0.0189 & -0.631 & -0.655 & -0.683 \\
0.5997 & 0.0000 & 0.089 & 0.226 & 0.371 & 0.0090 & 0.0141 & 0.0195 & -0.608 & -0.634 & -0.661 \\
\hline
\end{tabular}

Table 5. Values of excess-Gibb's free energy and excess-acoustic impedance at $2 \mathrm{MHz}$, $4 \mathrm{MHz}$ and $6 \mathrm{MHz}$

\begin{tabular}{|c|c|c|c|c|c|c|c|}
\hline \multicolumn{2}{|c|}{ Mole fraction } & \multicolumn{3}{|c|}{$\Delta \mathrm{G}^{\mathrm{E}}$} & \multicolumn{3}{|c|}{$\mathrm{Z}^{\mathrm{E}}$} \\
\hline $\mathrm{X}_{1}$ & $X_{3}$ & $\frac{\mathrm{x}}{2 \mathrm{MHz}}$ & $\frac{\mathrm{k} . J . \mathrm{n}}{4 \mathrm{MHz}}$ & $6 \mathrm{MHz}$ & $2 \mathrm{MHz}$ & $\frac{\mathrm{Kg} \cdot \mathrm{m}}{4 \mathrm{MHz}}$ & $6 \mathrm{MHz}$ \\
\hline 0.0000 & 0.6000 & 0.0562 & 0.0608 & 0.0694 & -0.045 & -0.050 & -0.061 \\
\hline 0.0999 & 0.4999 & 0.0721 & 0.0766 & 0.0854 & -0.048 & -0.053 & -0.064 \\
\hline 0.1998 & 0.4001 & 0.0761 & 0.0807 & 0.0890 & -0.050 & -0.056 & -0.066 \\
\hline 0.3001 & 0.3000 & 0.0822 & 0.0877 & 0.0960 & -0.051 & -0.058 & -0.068 \\
\hline 0.4000 & 0.1999 & 0.0854 & 0.0919 & 0.1002 & -0.053 & -0.060 & -0.071 \\
\hline 0.4998 & 0.1001 & 0.0903 & 0.0978 & 0.1063 & -0.054 & -0.063 & -0.073 \\
\hline 0.5997 & 0.0000 & 0.0910 & 0.0990 & 0.1075 & -0.056 & -0.065 & -0.076 \\
\hline
\end{tabular}

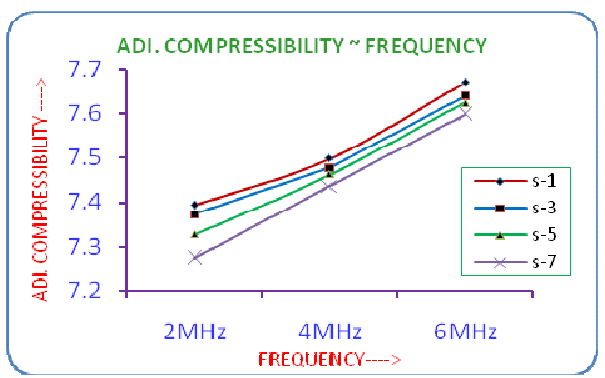

Figure 1. Variation of adi. Compressibility with frequency

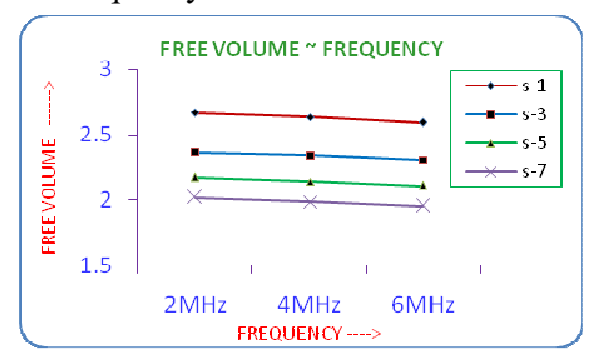

Figure 3. Variation of free volume with frequency

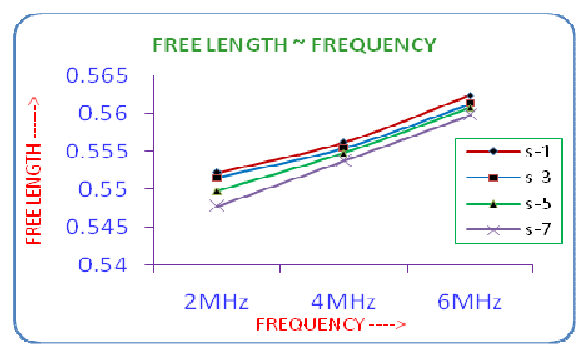

Figure 2. Variation of free length with frequency

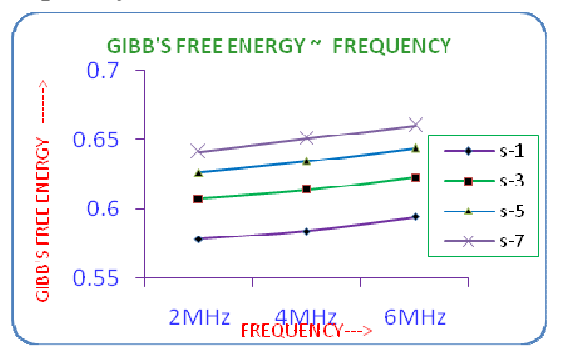

Figure 4. Variation of Gibb's free energy frequency 


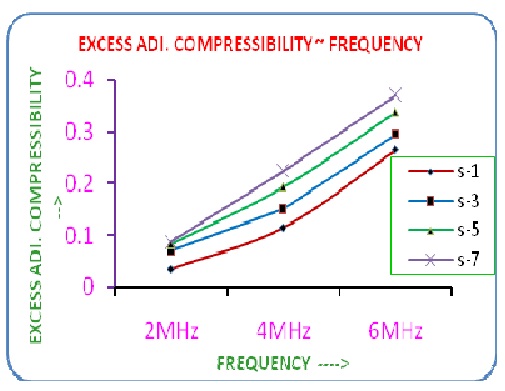

Figure 5. Variation of Acoustic impedance with coefficient frequency

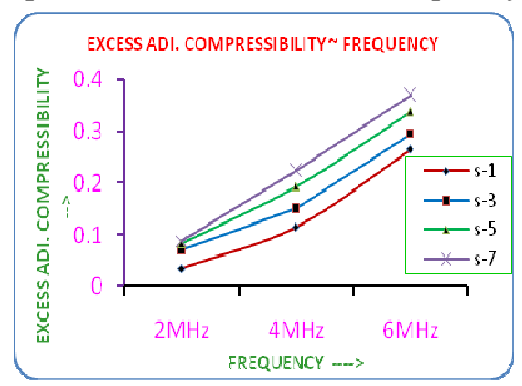

Figure 7: Variation of Excess adi. compressibility with Frequency

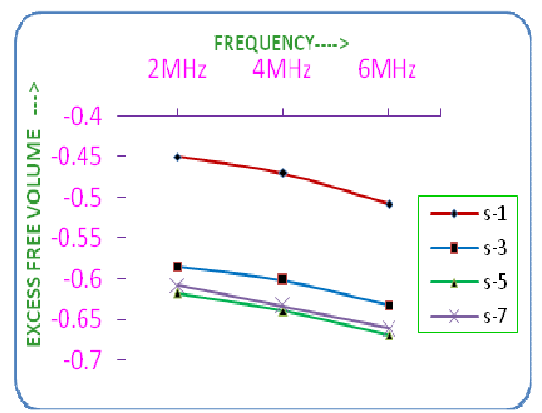

Figure 9. Variation of Excess free volume with frequency

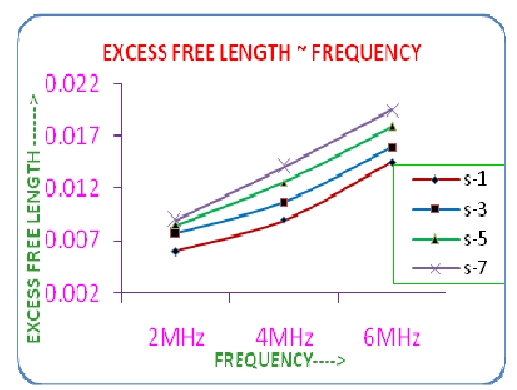

Figure 6. Variation of absorption with frequency

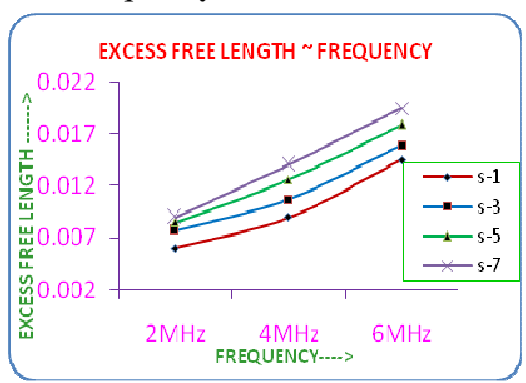

Figure 8. Variation of Excess free length with Frequency

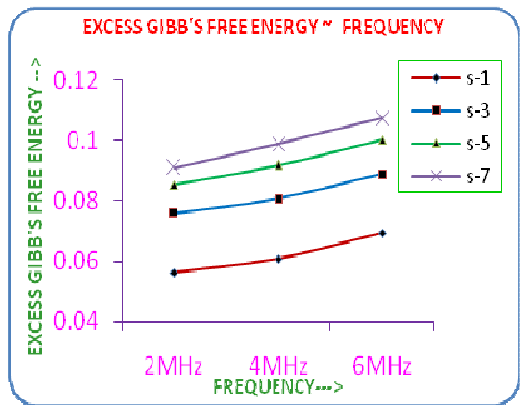

Figure 10. Variation of Excess Gibb's free energy

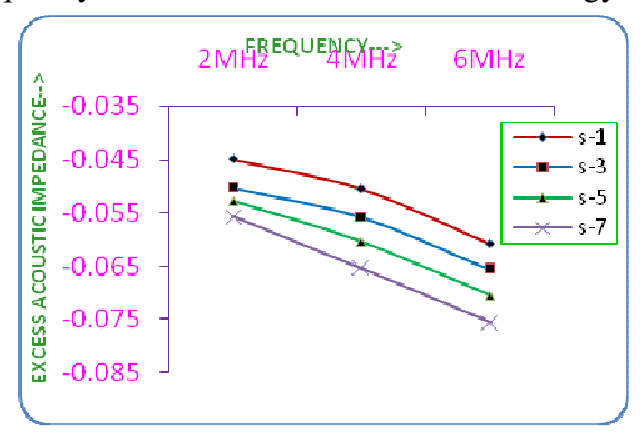

Figure 11. Variation of excess acoustic impedance with Frequency 
It is observed that, for a given concentration and temperature, ultrasonic velocity decreases with increasing frequency of the waves. Such a decrease in velocity is an indication of existence of molecular association between the components of the mixture. Again increasing trend of adiabatic compressibility is observed with increasing frequency. The ease, with which a medium can be compressed, is given by the compressibility. When frequency increases, the interaction between the molecules in the mixture changes causing a structural change and hence increase in adiabatic compressibility. Intermolecular free length depends on ' $\beta$ ' and shows a similar behavior as that of compressibility. Hence free length also increases but at a slower rate. On the basis of a model for sound propagation proposed by Eyring and Kincaid ${ }^{15}$ ultrasonic velocity should decrease if the intermolecular free length increases and vice versa.

Free volume is the average volume in which the center of a molecule can move due to the repulsion of the surrounding molecules. At constant concentration of components liquid and constant temperature, viscosity remains constant, hence

$$
\mathrm{V}_{\mathrm{f}} \propto \mathrm{U}^{3 / 2}
$$

Since velocity decreases as frequency increases, hence free volume also decreases.

With increase in frequency, concentration remains constant, velocity decreases and hence acoustic impedance decreases. This factor is governed by the inertial and elastic properties of the medium and hence supports the possibility of molecular interactions. Acoustic impedance is also used for assessing the absorption of sound in the medium. ' $\mathrm{Z}$ ' decreases with frequency.

Gibbs' free energy $(\Delta \mathrm{G})$ increases with frequency. The increase in ' $\Delta \mathrm{G}$ ' suggests shorter time for rearrangement of molecules. This also indicates existence of molecular association between the components of the liquid mixture ${ }^{16}$.

Absorption coefficient, which is a characteristic of the medium, increases with increase in frequency. This shows a similar behavior to that of the passage of electromagnetic waves through a conductor where the skin depth decreases with frequency of the incoming wave. Decrease in skin depth means increase in absorption.

In order to understand more about the nature of the interaction between the components of the liquid mixtures, it is necessary to discuss the same in terms of excess parameters rather than actual values. Weak interaction between unlike molecules gives in general positive excess values where as dipole-dipole, dipole-induced dipole, charge transfer and hydrogen bonding between unlike molecules give negative excess parameters ${ }^{17}$.

The value of excess intermolecular free length follows the same trend as that of excess value of adiabatic compressibility. Excess free volume is negative indicating strong interaction between the components of the mixture. Negative values of ' $\mathrm{V}_{\mathrm{f}}$ ' also indicate contraction in volume. This is obvious because of the relative small size of DMF and its linear aliphatic structure and also because of the spherical shape of pyridine molecules, which are tightly packed. The negative values of ' $\mathrm{V}_{\mathrm{f}}$ ' decreases as frequency increases, indicating that there exists a weak interaction between the DMF and pyridine molecules.

Excess value of Gibbs' free energy is positive and increases slowly. This is attributed to hydrogen bonding and charge transfer between the molecules of the mixture. As the mixture consists of polar and non-polar liquids, in addition to dipole-dipole interactions there is a dipole-induced dipole interaction, which leads to a negative excess value of acoustic impedance. As frequency increases, the compressibility increases indicating weakening of interaction between the molecules. This is also confirmed by gradual decrease in the negative value of acoustic impedance. 


\section{Conclusion}

From the above study it is seen that there exists molecular association between the components of the ternary mixture due to dipole-dipole, dipole-induced dipole, charge transfer and hydrogen bonding interactions, which varies with the change in the frequency of the ultrasonic wave.

\section{Acknowledgement}

We are thankful to the Management of Ajay Binay Institute of Technology, CDA, Cuttack, for providing the infrastructure as an initiation for the improvement of research activities in the Institute. We are also thankful to Department of Physics, Ravenshaw University for providing guidance through their experienced faculty members.

\section{References}

1. Kannappan V and Jaya Santhi R, Indian J Pure Appl Phys., 2005, 43(10), 750-754.

2. Kumar R, Jayakumar S and Kannappan V, Indian J Pure Appl Phys., 2008, 46, 169.

3. $\quad$ Ali A and Nain A K, Acoustics Lett., 1996, 19(9), 181-187.

4. Pandy J D, Pant N, Shukla A K, Sarika and Krishna V, Indian J Pure Appl Phys., 1989, 27, 246-248

5. Kannappan A N and Rajendiran V, Indian J Pure Appl Phys., 1992, 30, 240-242.

6. Thirumaran S and Ramesh J, Rasayan J Chem., 2009, 2(3), 733-739.

7. Ali A, Abida A K Nain and Hyder S, J Sol Chem., 2003, 32(10), 865-877.

8. Praharaj M.K, Satapathy A, Mishra S and Mishra P R, J Chem Phar Res., 2012, 4(4), 1910-1920.

9. Soni P L, A Textbook of Organic Chemistry, Sultan \& Chand sons Ltd., New Delhi, 1988.

10. Thirumaran S and Jayalakshmi K, Arc Appl Sc Res., 2009, 1(2), 24-31.

11. Varada Rajulu A, Sreenivasulu G and Raghuraman K S, Indian J Chem Tech., 1994, 1, 302-304.

12. Nikam P S and Hasan Mehdi, Asian J Chem., 1993, 5(2), 319-321.

13. Prasad N and Rajendra H, J Pure Appl Ultrason., 2003, 25, 25-30.

14. Das J K, Dash S K, Swain N and Swain B B, J Mole Liq., 1999, 81, 163-179.

15. Eyring H and Kincaid J F, J Chem Phys., 1938, 6, 620-629.

16. Reed C R, Prausnitz J M and Polling B E, The properties of gases and liquids, Mc Graw Hill, New York, 1998.

17. Fort R J and Moore W R, Trans Farad Soc., 1965, 61, 2102-2011. 